\title{
Research
}

\section{The cerebellum in maintenance of a motor skill: A hierarchy of brain and spinal cord plasticity underlies H-reflex conditioning}

\author{
Jonathan R. Wolpaw ${ }^{1}$ and Xiang Yang Chen \\ Laboratory of Nervous System Disorders Wadsworth Center, New York State Department of Health and State University of New \\ York at Albany, Albany, New York 12201-0509, USA
}

\begin{abstract}
Operant conditioning of the H-reflex, the electrical analog of the spinal stretch reflex, is a simple model of skill acquisition and involves plasticity in the spinal cord. Previous work showed that the cerebellum is essential for down-conditioning the H-reflex. This study asks whether the cerebellum is also essential for maintaining down-conditioning. After rats decreased the soleus $\mathrm{H}$-reflex over $50 \mathrm{~d}$ in response to the down-conditioning protocol, the cerebellar output nuclei dentate and interpositus (DIN) were ablated, and down-conditioning continued for 50-100 more days. In naive (i.e., unconditioned) rats, DIN ablation itself has no significant long-term effect on H-reflex size. During down-conditioning prior to DIN ablation, eight Sprague-Dawley rats decreased the H-reflex to $57 \%( \pm 4$ SEM) of control. It rose after ablation, stabilizing within $2 \mathrm{~d}$ at about $75 \%$ and remaining there until $\sim 40 \mathrm{~d}$ after ablation. It then rose to $\sim 130 \%$, where it remained through the end of study $100 \mathrm{~d}$ after ablation. Thus, DIN ablation in down-conditioned rats caused an immediate increase and a delayed increase in the H-reflex. The final result was an $\mathrm{H}$-reflex significantly larger than that prior to down-conditioning. Combined with previous work, these remarkable results suggest that the spinal cord plasticity directly responsible for down-conditioning, which survives only 5-10 d on its own, is maintained by supraspinal plasticity that survives $\sim 40 \mathrm{~d}$ after loss of cerebellar output. Thus, H-reflex conditioning seems to depend on a hierarchy of brain and spinal cord plasticity to which the cerebellum makes an essential contribution.
\end{abstract}

The challenge presented by the phenomena of learning has changed greatly in recent years. In the past, the challenge was to discover activity-dependent central nervous system (CNS) plasticity that might account for learning. Now, many different kinds of activity-dependent plasticity are known to occur throughout the CNS, and the challenge is to define exactly how this plasticity accounts for learned behavioral changes. The complexity and limited accessibility of the CNS and the fact that even simple learning involves multisite plasticity obscure the causal connections between this plasticity and behavioral changes (Wolpaw and Lee 1989; Carrier et al. 1997; Cohen et al. 1997; Lieb and Frost 1997; Thompson et al. 1997; Whalen and Pearson 1997; Hansel et al. 2001; King et al. 2001; Wolpaw and Tennissen 2001; Carey and Lisberger 2002; Medina et al. 2002; van Alphen and De Zeeuw 2002; Wolpaw 2002; Blazquez et al. 2003; Boyden et al. 2004; Broussard and Kassardjian 2004; Fanselow and Poulos 2005; Thompson 2005). The elucidation of these connections depends on experimental models derived from simple behaviors produced by defined and accessible neural circuitry.

The H-reflex, the electrical analog of the spinal stretch reflex (SSR), is perhaps the simplest and most accessible behavior of the vertebrate CNS. It is mediated mainly by a monosynaptic pathway consisting of the primary afferent neuron from the muscle spindle, its synapse on the $\alpha$-motoneuron in the spinal cord, and the motoneuron itself (Magladery et al. 1951; Matthews 1972; Henneman and Mendell 1981; Brown 1984). The SSR and the H-reflex, which change during early development, during skill acquisition later in life, and after spinal cord trauma, also change

\footnotetext{
'Corresponding author.

E-mail wolpaw@wadsworth.org; fax (518) 486-4910.

Article and publication are at http://www.learnmem.org/cgi/doi/10.1101/ Im.92706.
}

in response to an operant conditioning protocol (for review, see Wolpaw and Tennissen 2001). Monkeys, humans, rats, and mice exposed to this protocol can gradually decrease (i.e., downconditioning) or increase (i.e., up-conditioning) the SSR or the H-reflex (Wolpaw et al. 1983; Wolpaw 1987; Evatt et al. 1989; Chen and Wolpaw 1995; Wolf et al. 1995; Carp et al. 2005). According to a standard definition of "skill" as an adaptive behavior acquired through practice (Chen et al. 2005), operantly conditioned changes in the SSR and H-reflex constitute simple motor skills.

The learning of these simple skills is associated with multisite spinal cord plasticity (Wolpaw and Lee 1989; Carp and Wolpaw 1994, 1995; Feng-Chen and Wolpaw 1996; Wolpaw 1997; Carp et al. 2001; for review, see Wolpaw and Tennissen 2001; Wang et al. 2003, 2006). This learning requires the corticospinal tract (CST), and does not require other major descending pathways (Chen and Wolpaw 1997, 2002; Chen et al. 2002). The cerebellum is essential, at least for down-conditioning (Chen and Wolpaw 2005).

The present study set out to determine whether the cerebellum is also essential for the maintenance of down-conditioning that has already occurred. The results are quite remarkable and unexpected, and they provide important new insight into the CNS plasticity that underlies skill acquisition.

\section{Results}

Figure 1A shows the study protocol. Several weeks after each rat was implanted for study, control H-reflex size was measured for 10-17 d. Then, the rat was exposed to the down-conditioning protocol (i.e., the HRdown mode; see Materials and Methods) for $50 \mathrm{~d}$, the nuclei dentate and interpositus (DIN) were ablated, and HRdown exposure continued for 50 (three rats) or 100 (five rats) 
A

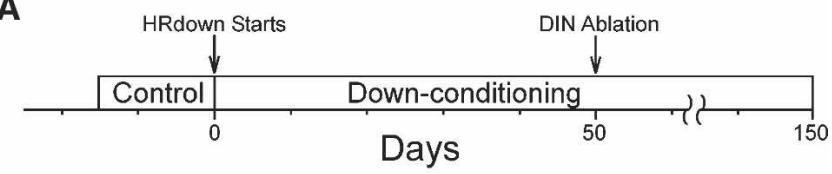

B
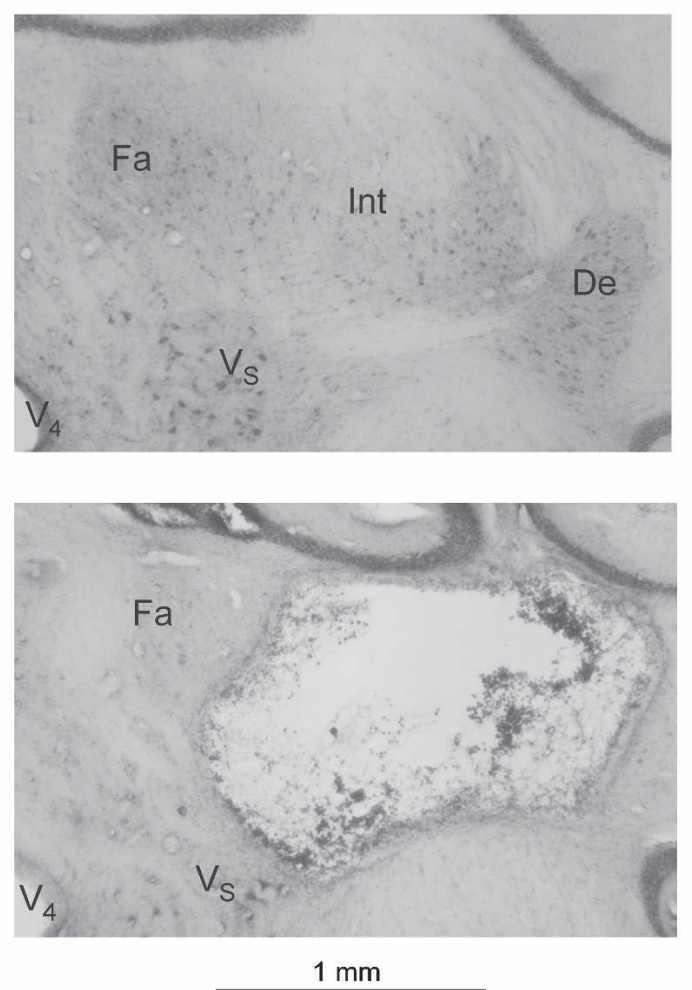

Figure 1. (A) Study protocol. At least $20 \mathrm{~d}$ after implantation surgery, control H-reflex size was measured over at least $10 \mathrm{~d}$, and the rats were exposed to down-conditioning (i.e., the HRdown mode) for $50 \mathrm{~d}$. Then the DIN were ablated, and down-conditioning continued through the end of data collection (i.e., for 50-100 more days). Background EMG amplitude and $\mathrm{M}$ response size were stable throughout. (B) DIN ablation. Photomicrographs of the right DIN area from a normal rat (top) and from a DIN rat (bottom). The dentate and interpositus nuclei are absent in the DIN rat. De indicates dentate nucleus; Fa, fastigial nucleus; Int, interpositus nucleus; $\mathrm{V}_{4}$, fourth ventricle; $\mathrm{V}_{\mathrm{S}}$, superior vestibular nucleus.

more days. Throughout the prolonged data collection from each rat, the number of trials per day (i.e., the number of H-reflex elicitations per day; see Materials and Methods), the background EMG at the time of H-reflex elicitation, and the $\mathrm{M}$ response size did not change from their values during the control period. Postmortem histological analysis showed that DIN ablation had been largely effective in all of the eight rats (see Materials and Methods). Figure $1 \mathrm{~B}$ illustrates the DIN ablation.

Figure 2A summarizes the results. It shows for all rats average $( \pm$ SEM $)$ H-reflex size (in percentage of control size) for each day throughout data collection.

\section{Effects of HRdown conditioning}

As Figure 2A shows, H-reflex size decreased steadily with HRdown exposure, reaching $57 \%$ ( \pm 4 SEM) of control for days $41-50$ of down-conditioning, just prior to DIN ablation. It did not vary significantly over these last 10 pre-ablation days $(P>0.1$, repeated-measures ANOVA). By the standard criterion for successful down-conditioning (i.e., a decrease to $\leq 80 \%$ of control $\mathrm{H}$ - reflex size) (Wolpaw et al. 1993; Chen and Wolpaw 1995), all eight rats were successful.

\section{Short-term effects of DIN ablation}

In the first $2 \mathrm{~d}$ after ablation, H-reflex size increased by $~ 50 \%$ of its control value and then fell back to a persistent increase of $\sim 20 \%$. Repeated-measures ANOVA applied to the first 10 postablation days showed significant variation from the average of the last 10 pre-ablation days $(P<0.001)$, and Dunnett's multiple comparisons test indicated that post-ablation days 1 and 2 differed significantly from the average of the last 10 pre-ablation days $(P<0.01$ for each) while none of days 3-10 did so $(P>0.05$ for each). A similar brief increase also occurs in the first $2 \mathrm{~d}$ after DIN ablation in naive rats (Chen and Wolpaw 2005), after cerebellar ablation in cats (Van Der Meulen and Gilman 1965; McLeod and Van Der Meulen 1967), and after mid-thoracic transection of the CST, the dorsal column ascending tract (DA), or the lateral column (LC) in naive or conditioned rats (Chen et al. 2001b, 2003; Chen and Wolpaw 2002). It is probably a nonspecific short-term effect of the surgery and/or the accompanying general anesthesia.

\section{Long-term effects of DIN ablation}

As Figure 2B shows, the average H-reflex in these downconditioned rats, which had fallen to $57 \%$ ( \pm 4 SEM) of control prior to DIN ablation, stabilized at $\sim 75 \%$ by $2 \mathrm{~d}$ after DIN ablation. It remained there until $40 \mathrm{~d}$ after ablation and then rose to $\sim 130 \%$ of control, where it remained through the end of data collection $100 \mathrm{~d}$ after DIN ablation. A statistical analysis that compared the entire course of H-reflex size following imposition of the HRdown mode to H-reflex size prior to down-conditioning confirmed this description. A repeated-measures ANOVA applied to the average $\mathrm{H}$-reflex values of the 10 -d periods from days $1-10$ through days 141-150 showed significant variation from the average of the last 10 control-mode days $(P<0.001)$. Dunnett's multiple comparisons test indicated that the average H-reflexes for days 11-20 through days 81-90 were significantly smaller that the average control H-reflex $(P<0.01$ for each period, except $P<0.05$ for days $71-80$ and days $81-90$ ), and that the average H-reflexes for days 101-110 through days 141-150 were significantly larger than the average control H-reflex $(P<0.01$ for each period).

Thus, although some of the H-reflex decrease produced by down-conditioning disappeared immediately after DIN ablation, a significant decrease below control survived until $40 \mathrm{~d}$ after ablation, after which it disappeared and was replaced by a significant increase above control that persisted through the end of data collection $100 \mathrm{~d}$ after DIN ablation. As described in Materials and Methods, the rats continued under the HRdown mode throughout the entire post-ablation period.

Figure 2B shows average daily H-reflexes from one rat for four representative days: one from the control-mode period prior to down-conditioning, one immediately prior to DIN ablation, one during the first $40 \mathrm{~d}$ after DIN ablation, and one near the end of data collection. Background EMG and M response size were stable throughout. These traces illustrate the decrease from control produced by down-conditioning, the early increase following DIN ablation that eliminates some but not all of the conditioned decrease, and the delayed larger increase that eliminates the conditioned decrease and results in an H-reflex larger than control.

\section{Comparison of the long-term effects of DIN ablation to those of other lesions}

The delayed increase $40 \mathrm{~d}$ after DIN ablation is probably the most striking feature of Figure 2, and was certainly unexpected. (In- 


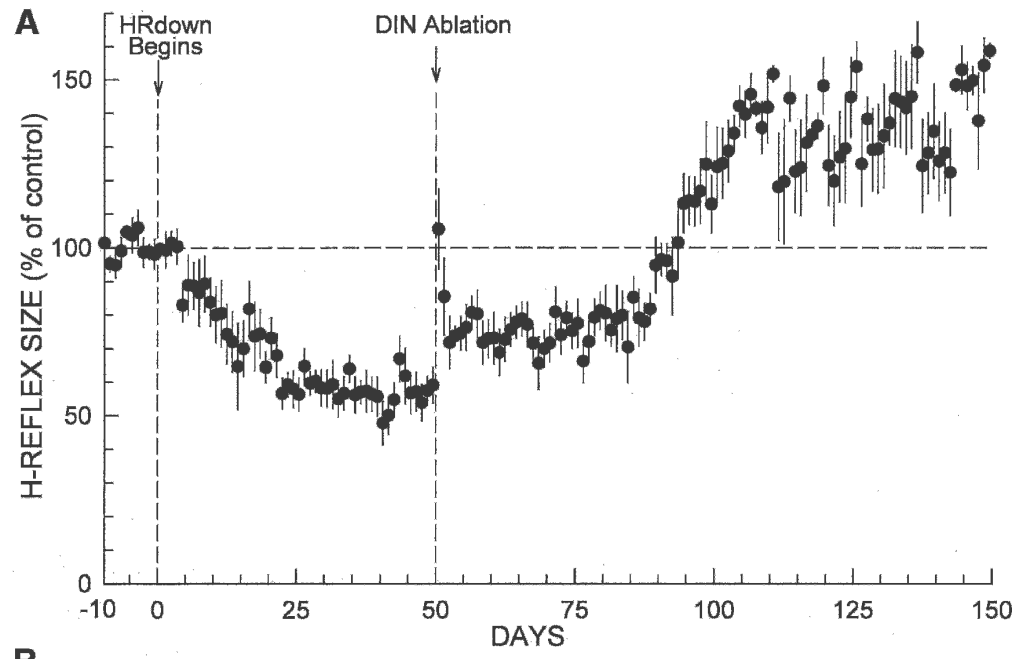

B
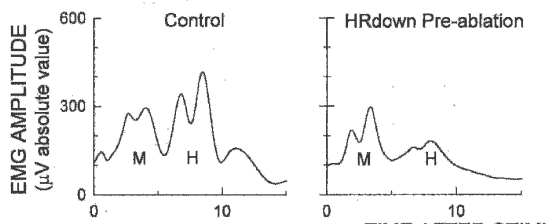

TIME AFTER STIMULUS (ms)

Figure 2. Effects of DIN ablation on H-reflex size in down-conditioned rats. $(A)$ Average H-reflex ( \pm SEM) (in percentage of average control size) for DIN rats for $150 \mathrm{~d}$ after imposition of the HRdown mode, with the time of DIN ablation shown. Background EMG amplitude and $\mathrm{M}$ response size were stable throughout. After DIN ablation, H-reflex size increased $\sim 20 \%$, remained there for $\sim 40 \mathrm{~d}$, and then rose to a value above its original control value and remained there through the end of data collection. (B) Daily average H-reflexes from a representative rat for a control day, a day just prior to DIN ablation, a day shortly after DIN ablation, and a day 100 d after DIN ablation. The early and delayed increases following DIN ablation are evident. Background EMG amplitude (i.e., the amplitude at time 0 ) and $\mathrm{M}$ response size were stable throughout.

deed, the initial plan was to continue data collection for only 50 $\mathrm{d}$ after DIN ablation. However, when the data from the first three rats revealed the delayed increase, data collection was extended for 50 more days for the other five rats.) Figure 3 compares the effects of DIN ablation on maintenance of down-conditioning with the effects of electrolytic mid-thoracic transection of the CST, the ipsilateral LC, or the DA, which are reported and fully described in earlier studies (Chen and Wolpaw 2002). All the lesions show the nonspecific transient increase in the first $2 \mathrm{~d}$. After this initial brief effect dissipates, neither LC transection (which interrupts the rubrospinal, reticulospinal, and vestibulospinal tracts) nor DA transection has any long-term effect. The H-reflex remains small for the next $50 \mathrm{~d}$ as exposure to the HRdown mode continues. In contrast, in 5-10 d after CST transection, the H-reflex rises to $125 \%( \pm 8)$, significantly greater than its original control size ( $P=0.04$, paired $t$-test), despite continued exposure to the HRdown mode. This high value, which is similar to the value reached $50 \mathrm{~d}$ after DIN ablation, remains through the end of data collection.

One-way ANOVA indicated that the final H-reflex values (i.e., average H-reflex size 91-100 d after DIN ablation and 41-50 $\mathrm{d}$ after LC, CST, or DA transection) differed among the four different lesions $(P<0.001)$. An all pair-wise multiple comparison procedure (Fisher LSD method) found that the final H-reflex of DIN rats differed significantly from that of DA or LC rats $(P<0.001$ for both) but not from that of CST rats $(P>0.7)$. CST transection and DIN ablation had similar final effects: an H-reflex larger than control $(125 \%$ [ \pm 8 SEM] and 130\% [ \pm 9 ] for CST and DIN ablation, respectively). The difference was that after CST transection, this high value was reached within $10 \mathrm{~d}$, while after
DIN ablation the H-reflex increased in two stages, and the second and larger stage was delayed for $40 \mathrm{~d}$.

\section{Discussion}

A previous study found that the cerebellum is essential for acquisition of an $\mathrm{H}$ reflex decrease in response to the HRdown mode (Chen and Wolpaw 2005). The present study evaluated the importance of the cerebellum in maintenance of an H-reflex decrease that has already occurred. As in the earlier study, cerebellar influence was removed by ablating the DIN. Interpretation of the results is simplified by the fact that DIN ablation alone, or DIN ablation followed by down-conditioning, has little or no long-term effect on H-reflex size (Chen and Wolpaw 2005). Furthermore, the ablation did not involve areas other than the DIN (e.g., Fig. 1B) (see Materials and Methods).

The results show that the cerebellum is needed for maintenance of downconditioning. Thus, it complements the earlier demonstration that the cerebellum is needed for acquisition of downconditioning (Chen and Wolpaw 2005), and it has parallel implications concerning the nature of the cerebellum's contribution. Given the fact that the CST is essential for maintenance and that other major descending pathways (including the rubrospinal tract) are not essential (Chen and Wolpaw 1997, 2002), it appears that the critical cerebellar contribution is output that ascends to sensorimotor cortex (the main origin of the CST) rather than output that descends to the spinal cord. This cerebellar output could be nonspecific, that is, it could simply support normal functioning of the sensorimotor cortex and thereby allow it to maintain the CST output essential for maintenance of the spinal cord plasticity that directly underlies the H-reflex decrease (Chen and Wolpaw 1997, 2002). Alternatively, this cerebellar output could be specific to down-conditioning of the soleus H-reflex; that is, it could specify the CST activity that maintains the spinal cord plasticity. In this case, the cerebellar output might reflect cerebellar plasticity similar to that thought to occur with vestibuloocular reflex (VOR) or eyeblink conditioning (e.g., as a result of the conjunction of activity in particular mossy and climbing fibers) (Yeo and Hesslow 1998; Ito 2000; Raymond and Lisberger 2000; Hansel et al. 2001; Carey and Lisberger 2002; Medina et al. 2002; Blazquez et al. 2003; Boyden et al. 2004; Bracha 2004; Broussard and Kassardjian 2004; Fanselow and Poulos 2005; Thompson 2005).

This maintenance study also provides two remarkable and important new pieces of information: DIN ablation after downconditioning has occurred leads eventually to an H-reflex that is significantly larger than is the control H-reflex prior to downconditioning, and this long-term effect of DIN ablation takes 50 $\mathrm{d}$ to occur and appears to develop in two stages, an immediate increase and a delayed increase.

\section{The larger final H-reflex}

The larger-than-control H-reflex evident from $100 \mathrm{~d}$ on (Fig. 2A) is paradoxical in that it occurs despite the continued imposition 


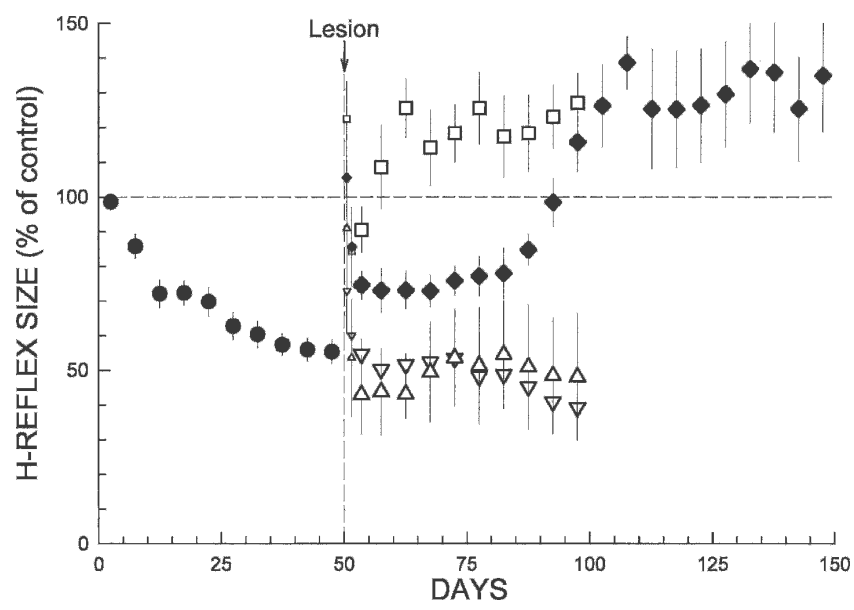

Figure 3. Comparison of the effects of different lesions on maintenance of H-reflex down-conditioning. Average $\mathrm{H}$-reflex sizes $( \pm \mathrm{SEM})$ for each 5 -d period for the first $50 \mathrm{~d}$ of down-conditioning for all 23 rats ( ) and for the next 50-100 d for the DIN ( $\bullet$, eight rats), CST ( $\square$, five rats), LC $(\triangle$, five rats), and DA $(\nabla$, five rats) groups (CST, LC, and DA data from Chen and Wolpaw 2002). For the $5 \mathrm{~d}$ immediately post-lesion, H-reflex sizes are shown for the first day and the second day (smaller symbols), and for the next $3 \mathrm{~d}$ together. All the lesions show a nonspecific transient increase in the first $2 \mathrm{~d}$. After this initial brief effect dissipates, neither LC nor DA transection has any long-term effect. In contrast, DIN ablation or CST transection results in an $\mathrm{H}$-reflex larger than control. The difference between the two lesions is that after CST transection the H-reflex increases within $10 \mathrm{~d}$, while after DIN ablation the H-reflex increases in two stages, and the second stage is delayed until $\sim 40 \mathrm{~d}$ after the lesion. As noted in the text, the HRdown mode remains in effect throughout.

of the HRdown mode (i.e., despite the fact that the rat continues to be rewarded for a smaller H-reflex). And, as noted above, it cannot be attributed to the DIN ablation itself, which has no significant long-term effect on H-reflex size (Chen and Wolpaw 2005). This big H-reflex is surprising, but not unprecedented. Four similarly unexpected increases have appeared in earlier studies of H-reflex conditioning. First, as Figure 3 shows, CST transection in down-conditioned rats leads to a similar increase over control (Chen and Wolpaw 2002). Second, in downconditioned monkeys, general anesthesia and spinal cord transection produce reflexes that are larger than expected on both the down-conditioned side and the contralateral side (Wolpaw and Lee 1989). Third, detailed analysis of the time course of $\mathrm{H}$ reflex down-conditioning in normal rats suggests that its beginning is associated with a small increase in H-reflex size that is obscured by the progressive development of the H-reflex decrease (Chen et al. 2001a). Fourth, and perhaps most remarkable, is the increase found in recent studies of contralateral sensorimotor cortex (cSMC) ablation. When rats with cSMC ablation are exposed to the control mode, the H-reflex does not change (Chen et al. 2004; data not shown). However, when these cSMC rats are exposed to the HRdown mode, the H-reflex increases (rather than decreases) over $6 \mathrm{~d}$ (Chen et al. 2004; data not shown).

These previous observations and the increase seen in this study are consistent with other data indicating that H-reflex conditioning is associated with activity-dependent plasticity at multiple sites (Wolpaw and Lee 1989; Carp and Wolpaw 1994, 1995; Feng-Chen and Wolpaw 1996; Wolpaw 1997; Carp et al. 2001; Wang et al. 2003, 2006; for review, see Wolpaw and Tennissen 2001). While some of this plasticity appears responsible for the conditioned H-reflex change (i.e., primary plasticity), other plasticity may ensure the preservation of older skills such as locomotion (i.e., compensatory plasticity), or may simply reflect reactive downstream effects caused by changes in activity due to primary or compensatory plasticity (i.e., reactive plasticity) (Wolpaw and Lee 1989; Wolpaw and Carp 1990; Wolpaw 1997; Wolpaw and Tennissen 2001).

The plasticity responsible for the paradoxical long-term $\mathrm{H}$ reflex increase in Figure 2A, which is likely to be either compensatory or reactive, could be located in the spinal cord or in the brain. The paradoxical increase with anesthesia and spinal cord transection in monkeys is certainly of spinal origin: It is evident only when the spinal cord is isolated from the brain (Wolpaw and Lee 1989). On the other hand, the increase after CST transection (Fig. 3) could be an effect of supraspinal plasticity that operates through non-CST pathways and is normally opposed by CST influence (so that it only emerges following CST transection). In this case, LC transection in down-conditioned rats might be expected to produce a rapid further decrease in H-reflex size. Figure 3 does suggest that LC transection in downconditioned rats may be followed by some additional decrease (for full data, see Chen and Wolpaw 2002).

All of the other four paradoxical increases described above occur immediately or in a few days. This suggests that the increase above control seen in Figure $2 \mathrm{~A}$ is the same increase evident by $2 \mathrm{~d}$ after DIN ablation, and that for the next $40 \mathrm{~d}$ it coexists with the H-reflex decrease produced by downconditioning. It is only $40-50 \mathrm{~d}$ after DIN ablation, when the H-reflex decrease finally disappears, that the H-reflex actually becomes larger than control.

\section{The delayed H-reflex increase}

As the previous section indicates, the larger final H-reflex, although unexpected, is not unprecedented. In contrast, the delayed increase also evident in Figure $2 \mathrm{~A}$ is both unexpected and unprecedented. Following the immediate increase after DIN ablation, the H-reflex size remains stable at a level significantly below its original control size for $\sim 40 \mathrm{~d}$. It then rises to its final high value, which is comparable to the value reached by $10 \mathrm{~d}$ after CST transection (Fig. 3). The delayed increase is particularly striking in that it is evident in the averaged data from all the rats and is not accompanied by an increase in the standard error (which only increases past day 100 when the number of rats falls from eight to five and the individual H-reflex values become considerably higher). Nothing like this delayed increase has occurred before in extensive explorations of H-reflex and SSR conditioning in monkeys and rats (Wolpaw and Tennissen 2001; for review, see Wolpaw 2001). A similar delayed increase does not occur after DIN ablation or other lesions in naive rats, or after CST, DA, or LC transection in down-conditioned or upconditioned rats (Chen and Wolpaw 1997, 2002; Chen et al. 2001b, 2002, 2003).

The 40-d stability followed by the increase, occurring in the context of the continued presence of the HRdown mode, implies that the plasticity responsible for a smaller H-reflex survives DIN ablation for $\sim 40 \mathrm{~d}$ and then deteriorates. This plasticity is unlikely to be spinal: The rapid effect of CST transection, illustrated in Figure 3, indicates that the spinal plasticity that is directly responsible for the smaller H-reflex can survive only 5-10 d on its own. Thus, the plasticity that deteriorates after $40 \mathrm{~d}$ is more likely to be supraspinal and to be responsible for the CST influence that maintains the spinal cord plasticity. Plasticity in sensorimotor cortex and associated regions, which occurs in many physiological and pathological situations (Nudo 2003; Siebner and Rothwell 2003; Field-Fote 2004; Luft et al. 2005; Sawaki 2005), is a strong possibility, and the pathways mediating cerebrocerebellar interactions, which are important for a variety of motor behaviors (Glickstein and Yeo 1990; Leiner et al. 1991; Raymond et al. 1996; Houk 1997; Schmahmann and Pandyat 1997; Desmond and Fiez 1998; Middleton and Strick 1998; Thach 1998; Holdefer 
et al. 2000; Mandolesi et al. 2001; Schwarz and Mock 2001; Schwarz and Welsh 2001; Molinari et al. 2002; Andre and Arrogi 2003; Mori et al. 2004; Nitschke et al. 2005), are likely to support the maintenance of this plasticity.

DIN ablation had no lasting effect on animal well-being, gross motor behavior, or activity level: Rats gained weight, walked normally, and satisfied the background EMG requirement (see Materials and Methods) with the same daily frequency as before ablation. Combined with the long delay in the loss of down-conditioning, these observations suggest that the effect of DIN ablation on maintenance of down-conditioning is not due to a nonspecific impairment of cortical function. It suggests that cerebellar influence (and perhaps cerebellar plasticity; see above) produced by the reward contingency is essential for the longterm (i.e., $>40 \mathrm{~d}$ ) maintenance of the supraspinal plasticity. The fact that the full effect of DIN ablation was not evident for $50 \mathrm{~d}$ implies that investigations of cerebellar participation in learning and memory may require data collection over very prolonged periods.

\section{A hierarchy of plasticity}

H-reflex down-conditioning clearly changes the spinal cord, and the results of descending pathway transections indicate that this spinal cord plasticity is created by CST activity and can survive only 5-10 d without it (Chen and Wolpaw 1997, 2002). The results of the present study imply that this CST activity depends on supraspinal plasticity that can survive $\sim 40 \mathrm{~d}$ without the cerebellum. Thus, H-reflex down-conditioning appears to depend on a hierarchy of brain and spinal plasticity. The spinal plasticity directly underlies the conditioned H-reflex change and can survive on its own for only a short time (5-10 d). It is maintained by supraspinal plasticity that is created by the reward contingency. As discussed in detail previously (Carp and Wolpaw 1994; Halter et al. 1995), the spinal cord plasticity includes a positive shift in motoneuron firing threshold that may be due to a comparable shift in sodium channel activation voltage, and recent data suggest that the shift might be mediated through metabotropic GABA-B input to the motoneuron (Pillai et al. 2004; Wang et al. $2004,2006)$. The nature and site(s) of the supraspinal plasticity are unknown, although sensorimotor cortex is a likely candidate.

The long-term paradoxical increase evident in Figure 2A implies that this hierarchy is associated with additional spinal or supraspinal plasticity, which, as noted above, may serve to preserve previously acquired skills. When the hierarchy is intact, the effect of this additional plasticity on the H-reflex is not evident. It becomes evident only when the hierarchy is disrupted (e.g., by DIN ablation [present study], CST transection [Chen and Wolpaw 2002], or complete spinal cord transection [Wolpaw and Lee 1989]).

\section{Conclusions}

Earlier studies implied that the spinal cord plasticity underlying down-conditioning is created and maintained by CST activity, and that the development of this activity requires the cerebellum. The present study shows that the long-term maintenance of the CST activity that maintains the spinal cord plasticity also requires the cerebellum, and it thereby implies the existence of supraspinal plasticity that requires the cerebellum for its longterm survival. H-reflex down-conditioning appears to depend on a hierarchy of plasticity, and the cerebellum appears to be essential for the creation and maintenance of this hierarchy.

\section{Materials and Methods}

The study used eight adult Sprague-Dawley rats (three females weighing 266-299 g initially and five males weighing 342-586 g initially). All procedures satisfied the Guide for the Care and Use of Laboratory Animals of the Institute of Laboratory Animal Resources, Commission on Life Sciences, National Research Council (National Academy Press, Washington, DC, 1996) and had been reviewed and approved by the Institutional Animal Care and Use Committee of the Wadsworth Center. The H-reflex conditioning and DIN ablation protocols, which are described in detail elsewhere (Wolpaw and Herchenroder 1990; Chen and Wolpaw 1995, 2005), are summarized here.

\section{The H-reflex conditioning protocol}

Each rat was implanted with chronic stimulating and recording electrodes in the right hindlimb under general anesthesia (ketamine $\mathrm{HCl}[80 \mathrm{mg} / \mathrm{kg}]$ and xylazine $[10 \mathrm{mg} / \mathrm{kg}]$; both intraperitoneally [i.p.]). To elicit the H-reflex, a nerve stimulating cuff was placed on the right posterior tibial nerve just above the triceps surae branches. To record soleus EMG, fine-wire electrodes were placed in the right soleus muscle. The Teflon-coated wires from the nerve cuff and the muscle went subcutaneously to a connector plug on the head. Data collection started at least $20 \mathrm{~d}$ later. During data collection, the rat lived in a standard rat cage with a flexible cable attached to the head plug. The cable allowed the animal to move freely about the cage and conveyed the wires from the electrodes to an electronic swivel above the cage, from which they went to an EMG amplifier and a nerve-cuff stimulation unit. All animals had full access to water and food, except that, during H-reflex conditioning, they received food mainly through the task described below. Animal well-being was carefully checked several times every day, and body weight was measured every week. Laboratory lights were dim from $2100-0600 \mathrm{~h}$ each day.

A computer system constantly monitored EMG from soleus muscle and controlled the nerve cuff stimulus. Whenever the absolute value (i.e., equivalent to the full-wave rectified value) of the background (i.e., ongoing) EMG remained within a defined range (typically $1 \%-2 \%$ of maximum possible EMG as determined by maximum $\mathrm{M}$ response) for a randomly varying 2.3 - to 2.7 -sec period, a stimulus pulse (typically $0.5 \mathrm{msec}$ in duration) was given by the nerve cuff. This criterion ensured that background EMG at the time of H-reflex elicitation was the same throughout data collection. Pulse amplitude was initially set just above M-response threshold and then continuously and automatically adjusted to keep M-response size unchanged. Thus, both the background EMG (reflecting soleus motoneuron tone at the time of H-reflex elicitation) and the M-response (reflecting the effective strength of the nerve cuff stimulus) were stable throughout the entire period of data collection. Under the control mode, the computer simply measured the absolute value of soleus EMG for 50 msec after nerve stimulation and determined H-reflex size. Under the HRdown mode, it also gave a food reward 200 msec after nerve stimulation if EMG amplitude in the $\mathrm{H}$ reflex interval (i.e., typically $6.0-10.0 \mathrm{msec}$ after stimulation) was below a criterion value (which was initially defined on the basis of the control-mode data so as to reward the smallest $20 \%-30 \%$ of the rat's H-reflexes, and was subsequently adjusted as needed to maintain this reward percentage). In the course of its normal activity, the animal usually satisfied the background EMG requirement, and thus received nerve stimulation, 3200-8900 times per day. H-reflex size was measured as average EMG amplitude in the H-reflex interval minus average background EMG amplitude and was expressed in units of average background EMG amplitude. As indicated in the Results, each rat's number of trials per day, background EMG amplitude, and $\mathrm{M}$ response size were stable throughout data collection.

\section{DIN ablation and post-ablation animal care and well-being}

Bilateral ablation of the DIN (Voogd 1995; Voogd and Glickstein 1998) was performed electrolytically with a platinum/iridium electrode (monopolar, 0.125-mm diameter, $0.100 \mathrm{~mm}$ exposed). (Ablation was bilateral so that, if the results were negative [i.e., if conditioning was maintained in the DIN rats], they would clearly

\section{Learning \& Memory \\ www.learnmem.org}


rule out an essential role for the cerebellum.) The rat was anesthetized as described above and placed in a stereotaxic frame. Two holes (1-mm diameter) were made in the skull on each side. For dentate ablation, the electrode was positioned vertical to the plane defined by the interaural line and the midline with its tip on the surface of the brain $2.70 \mathrm{~mm}$ caudal to the interaural line and $2.72 \mathrm{~mm}$ lateral to the midline, and the tip was then inserted vertically $4.10 \mathrm{~mm}$ into the brain (Paxinos and Watson 1986; Kruger et al. 1995; Chen and Wolpaw 2005). For interpositus ablation, the electrode was positioned vertical to the plane defined by the interaural line and the midline with its tip on the surface of the brain $2.70 \mathrm{~mm}$ caudal to the interaural line and $1.72 \mathrm{~mm}$ lateral to the midline, and the tip was then inserted vertically $4.50 \mathrm{~mm}$ into the brain. Each ablation was achieved by passing AC current $(0.2-0.3 \mathrm{~mA}, 5-\mathrm{Hz}$ sine wave) for $7 \mathrm{~min}$. The electrode was then removed, and the hole was filled with bone wax. After both nuclei were ablated on both sides, muscle and skin were sutured in layers.

Immediately after ablation, the rat was put under a heat lamp and given an analgesic (Demerol, $0.2 \mathrm{mg}$, intramuscularly [i.m.]). Once awake, it got a second dose of analgesic and was returned to its cage. Rats that ate poorly in the first few postablation days were fed manually with water-soaked chow and a dietary supplement (Nutri-Cal). All rats resumed eating normally within $5 \mathrm{~d}$ and were healthy and active throughout the remainder of the study. Body weight fell $4 \%-17 \%$ in the first postablation week, and regained its pre-ablation level in 11-43 d. Every rat gained weight over the period of study. For all rats, weight increased from 265-586 g at the beginning to 351-673 g at the end. Locomotion, which was frequently awkward and poorly balanced immediately after DIN ablation, appeared normal in $3-4$ wk or less.

\section{Histology}

At the end of data collection, each rat received an overdose of sodium pentobarbital (i.p.) and was then perfused through the heart with saline followed by 3\% paraformaldehyde and 1\% glutaraldehyde in $0.1 \mathrm{M}$ phosphate buffer (pH 7.3). The EMG electrodes, nerve cuff, and tibial nerve were examined, and the right and left soleus muscles were removed and weighed (as percentage of body weight). Their weights were symmetrical and did not differ significantly from normal.

The brain was removed and the area including the cerebellum was stored in $10 \%$ sucrose in $0.2 \mathrm{M}$ phosphate buffer $(\mathrm{pH}$ 7.3). Transverse $100-\mu \mathrm{m}$ serial sections were stained with $1 \%$ neutral red. In the sections including the DIN, we counted, on right and left sides, the number of DIN cells with diameters $\geq 10 \mu \mathrm{m}$. Four normal (i.e., unablated) rats were similarly studied (Chen and Wolpaw 2005). The number of DIN cells remaining on each side of each DIN rat was calculated as the percentage of the average number of cells on each side of the normal rats.

Normal rats averaged 2879 ( \pm 91 SD) DIN cells on each side, with no significant difference between right and left sides $(P>0.05$ by paired $t$-test) (Chen and Wolpaw 2005). DIN ablation was largely effective in all eight of the DIN rats. Total cell counts averaged $17 \%( \pm 10$ SD) (range 1\%-30\%) of normal. The right DIN averaged 15\% ( \pm 10 SD) (range 1\%-26\%) and the left DIN averaged 18\% ( \pm 10 SD) (range 2\%-33\%). Figure 1B shows transverse sections from a normal rat and a DIN rat. Beyond the nearly total loss of the DIN nuclei, DIN rats showed little or no damage other than the narrow tract of the electrode through the cerebellum. Thus, the effects of DIN ablation were almost certainly due to DIN loss rather than to collateral damage to other structures.

\section{Data analysis}

To assess short-term effects on H-reflex size of DIN ablation in these down-conditioned rats (i.e., Figure 2), a repeated-measures ANOVA was used to compare the first 10 post-ablation days to the last 10 pre-ablation days. If an effect was found, Dunnett's multiple comparisons method was used to identify post-ablation days that differed significantly from the average of the last 10 pre-ablation days. To assess the long-term effects of DIN ablation, a similar procedure was used to compare the average H-reflex size for each 10-d period after onset of the HRdown mode to the average H-reflex size for the last 10 control days. (For the first 10 -d period after DIN ablation, the average for days 3-10 was used to exclude the nonspecific transient increase seen in days 1-2.) If an effect was found, Dunnett's multiple comparisons method was used to identify those 10-d periods that differed significantly from the average of the last 10 control days. In addition, to compare the effects of different lesions (i.e., DIN ablation, LC, CST, or DA transection) on maintenance of H-reflex down-conditioning, a one-way ANOVA followed by an all pairwise multiple comparison procedure (Fisher LSD method) was used to compare the final H-reflex sizes (i.e., average H-reflex size for days 91-100 after DIN ablation and days 41-50 after LC, CST, or DA transection).

\section{Acknowledgments}

We thank Lu Chen, Rongliang Liu, and Scott Parsons for excellent technical assistance, and Jonathan S. Carp, Dennis J. McFarland, and Elizabeth Winter Wolpaw for valuable comments on the manuscript. This work was supported in part by grants from the National Institutes of Health HD36020 (X.Y.C.) and NS22189 (J.R.W.), the Christopher Reeve Paralysis Foundation (X.Y.C.), and the International Spinal Research Trust (J.R.W.).

\section{References}

Andre, P. and Arrogi, P. 2003. Hipnic modulation of cerebellar information processing: Implications the cerebro-cerebellar dialogue. Cerebellum 2: 84-95.

Blazquez, P.M., Hirata, Y., Heiney, S.A., Green, A.M., and Highstein, S.M. 2003. Cerebellar signatures of vestibulo-ocular reflex motor learning. J. Neurosci. 23: 9742-9751.

Boyden, E.S., Katoh, A., and Raymond, J.L. 2004. Cerebellum-dependent learning: The role of multiple plasticity mechanisms. Annu. Rev. Neurosci. 27: 581-609.

Bracha, V. 2004. Role of the cerebellum in eyeblink conditioning. Prog. Brain Res. 143: 331-339.

Broussard, D.M. and Kassardjian, C.D. 2004. Learning in a simple motor system. Learn. Mem. 11: 127-136.

Brown, W.F. 1984. The physiological and technical basis of electromyography. Butterworths, Boston.

Carey, M.R. and Lisberger, S.G. 2002. Embarrassed, but not depressed: Eye opening lessons for cerebellar learning. Neuron 35: 223-226.

Carp, J.S. and Wolpaw, J.R. 1994. Motoneuron plasticity underlying operantly conditioned decrease in primate H-reflex. J. Neurophysiol. 72: $431-442$.

. 1995. Motoneuron properties after operantly conditioned increase in primate H-reflex. J. Neurophysiol. 73: 1365-1373.

Carp, J.S., Chen, X.Y., Sheikh, H., and Wolpaw, J.R. 2001. Operant conditioning of rat H-reflexes affects motoneuron axonal conduction velocity. Exp. Brain Res. 136: 269-273.

Carp, J.S., Tennissen, A.M., Chen, X.Y., and Wolpaw, J.R. 2005. H-reflex conditioning in the mouse: Initial studies. Abstract Viewer/Itinerary Planner, Program No. 175.2. Society for Neuroscience, Washington, DC.

Carrier, L., Brustein, S., and Rossignol, S. 1997. Locomotion of the hindlimbs after neurectomy of ankle flexors in intact and spinal cats. I. Neurophysiol. 77: 1979-1993.

Chen, X.Y. and Wolpaw, J.R. 1995. Operant conditioning of H-reflex in freely moving rats. J. Neurophysiol. 73: 411-415.

. 1997. Dorsal column but not lateral column transection prevents down conditioning of H-reflex in rats. J. Neurophysiol. 78: $1730-1734$.

. 2002. Probable corticospinal tract control of spinal cord plasticity in rats. J. Neurophysiol. 87: 645-652.

. 2005. Ablation of cerebellar nuclei prevents H-reflex down-conditioning in rats. Learn. Mem. 12: 248-254.

Chen, X.Y., Chen, L., and Wolpaw, J.R. 2001a. Time course of H-reflex conditioning in the rat. Neurosci. Lett. 302: 85-88.

Chen, X.Y., Feng-Chen, K.C., Chen, L., Stark, D.M., and Wolpaw, J.R. 2001b. Short-term and medium-term effects of spinal cord tract transections on soleus H-reflex in freely moving rats. J. Neurotrauma 18: 313-327.

Chen, X.Y., Carp, J.S., Chen, L., and Wolpaw, J.R. 2002. Corticospinal 
tract transection prevents operantly conditioned H-reflex increase in rats. Exp. Brain Res. 144: 88-94.

Chen, X.Y., Chen, L., and Wolpaw, J.R. 2003. Conditioned H-reflex increase persists after transection of the main corticospinal tract in rats. J. Neurophysiol. 90: 3572-3578.

- 2004. Contralateral sensorimotor cortex ablation in rats impairs H-reflex up-conditioning and reverses the effect of down-conditioning. Abstract Viewer/Itinerary Planner, Program No. 417.4. Society for Neuroscience, Washington, DC.

Chen, Y., Chen, X.Y., Jakeman, L.B., Schalk, G., Stokes, B.T., and Wolpaw, J.R. 2005. The interaction of a new motor skill and an old one: H-reflex conditioning and locomotion in rats. J. Neurosci. 25: 6898-6906.

Cohen, T.E., Kaplan, S.W., Kandel, E.R., and Hawkins, R.D. 1997. A simplified preparation for relating cellular events to behavior: Mechanisms contributing to habituation, dishabituation, and sensitization of the Aplysia gill-withdrawal reflex. J. Neurosci. 17: 2886-2899.

Desmond, J.E. and Fiez, J.A. 1998. Neuroimaging studies of the cerebellum: Language, learning and memory. Trends Cogn. Sci. 2: $355-362$.

Evatt, M.L., Wolf, S.L., and Segal, R.L. 1989. Modification of human spinal stretch reflexes: Preliminary studies. Neurosci. Lett. 105: 350-355.

Fanselow, M.S. and Poulos, A.M. 2005. The neuroscience of mammalian associative learning. Annu. Rev. Psychol. 56: 207-234.

Feng-Chen, K.C. and Wolpaw, J.R. 1996. Operant conditioning of H-reflex changes synaptic terminals on primate motoneurons. Proc. Natl. Acad. Sci. 93: 9206-9211.

Field-Fote, E.C. 2004. Electrical stimulation modifies spinal and cortical neural circuitry. Exerc. Sport Sci. Rev. 32: 155-160.

Glickstein, M. and Yeo, C. 1990. The cerebellum and motor learning. J. Cogn. Neurosci. 2: 69-80.

Halter, J.A., Carp, J.S., and Wolpaw, J.R. 1995. Operantly conditioned motoneuron plasticity: Possible role of sodium channels. $J$. Neurophysiol. 74: 867-871.

Hansel, C., Linden, D.J., and D'Angelo, E. 2001. Beyond parallel fiber LTD: The diversity of synaptic and non-synaptic plasticity in the cerebellum. Nat. Neurosci. 4: 467-475.

Henneman, E. and Mendell, L.M. 1981. Functional organization of motoneuron pool and its inputs. In Handbook of physiology: The nervous system, pp. 423-507. American Physiological Society, Bethesda, MD.

Holdefer, R.N., Miller, L.E., Chen, L.L., and Houk, J.C. 2000. Functional connectivity between cerebellum and primary motor cortex in the awake monkey. J. Neurophysiol. 84: 585-590.

Houk, J.C. 1997. On the role of the cerebellum and basal ganglia in cognitive signal processing. Prog. Brain Res. 114: 543-552.

Ito, M. 2000. Mechanisms of motor learning in the cerebellum. Brain Res. 886: 237-245.

King, D.A.T., Krupa, D.J., Foy, M.R., and Thompson, R.F. 2001. Mechanisms of neuronal conditioning. Int. Rev. Neurobiol. 45: 313-337.

Kruger, L., Saporta, S., and Swanson, L.W. 1995. Photographic atlas of the rat brain. Cambridge University Press, Cambridge, UK.

Leiner, H.C., Leiner, A.L., and Dow, R.S. 1991. The human cerebro-cerebellar system: Its computing, cognitive, and language skills. Behav. Brain Res. 44: 113-128.

Lieb, J.R. and Frost, W.N. 1997. Realistic simulation of the Aplysia siphon-withdrawal reflex circuit: Roles of circuit elements in producing motor output. J. Neurophysiol. 77: 1249-1268.

Luft, A.R., Manto, M.-U., and Ben Taib, N.O. 2005. Modulation of motor cortex excitability by sustained peripheral stimulation: The interaction between the motor cortex and the cerebellum. Cerebellum 4: 90-96.

Magladery, J.W., Porter, W.E., Park, A.M., and Teasdall, R.D. 1951. Electrophysiological studies of nerve and reflex activity in normal man, IV: The two-neuron reflex and identification of certain action potentials from spinal roots and cord. Bull. Johns Hopkins Hosp. 88: 499-519.

Mandolesi, L., Leggio, M.G., Graziano, A., Neri, P., and Petrosini, L. 2001. Cerebellar contribution to spatial event processing: Involvement in procedural and working memory components. Eur. J. Neurosci. 14: 2011-2022.

Matthews, P.B.C. 1972. Mammalian muscle receptors and their central actions. Williams \& Wilkins, Baltimore.

McLeod, J.G. and Van Der Meulen, J.P. 1967. Effect of cerebellar ablation on the $H$ reflex in the cat. Arch. Neurol 16: 421-432.

Medina, J.F., Repa, J.C., Mauk, M.D., and LeDoux, J.E. 2002. Parallels between cerebellum- and amygdala-dependent conditioning. Nat. Rev. Neurosci. 3: 122-131.

Middleton, F.A. and Strick, P.L. 1998. Cerebellar output: Motor and cognitive channels. Trends Cogn. Sci. 2: 348-354.

Molinari, M., Filippini, V., and Leggio, M.G. 2002. Neuronal plasticity of interrelated cerebellar and cortical networks. Neuroscience 111: 863-870.

Mori, S., Nakajima, K., Mori, F., and Matsuyama, K. 2004. Integration of multiple motor segments for the elaboration of locomotion: Role of the fastigial nucleus of the cerebellum. Prog. Brain Res. 143: 341-351.

Nitschke, M.F., Arp, T., Stavrou, G., Erdmann, C., and Heide, W. 2005. The cerebellum in the cerebro-cerebellar network for the control of eye and hand movements: An fMRI study. Prog. Brain Res.

148: $151-164$

Nudo, R.J. 2003. Adaptive plasticity in motor cortex: Implications for rehabilitation after brain injury. J. Rehabil. Med. 41: 7-10.

Paxinos, G. and Watson, C. 1986. The rat brain in stereotaxic coordinates, 2nd ed. Academic Press, San Diego.

Pillai, S.D., Wang, Y., Wolpaw, J.R., and Chen, X.Y. 2004. H-reflex down-conditioning in rats increases number and size of GABAergic terminals on soleus motoneurons. Abstract Viewer/Itinerary Planner, Program No. 417.2. Society for Neuroscience, Washington, DC.

Raymond, J.L. and Lisberger, S.G. 2000. Hypotheses about the neural trigger for plasticity in the circuit for the vestibulo-ocular reflex. Prog. Brain Res. 124: $235-246$.

Raymond, J.L., Lisberger, S.G., and Mauk, M.D. 1996. The cerebellum: A neuronal learning machine? Science 272: 1126-1131.

Sawaki, L. 2005. Use-dependent plasticity of the human motor cortex in health and disease. IEEE Eng. Med. Biol. 36-39.

Schmahmann, J.D. and Pandyat, D.N. 1997. The cerebrocerebellar system. Int. Rev. Neurobiol. 41: 31-60.

Schwarz, C. and Mock, M. 2001. Spatial arrangement of cerebro-pontine terminals. J. Comp. Neurol. 435: 418-432.

Schwarz, C. and Welsh, J.P. 2001. Dynamic modulation of mossy fiber system throughput by inferior olive synchrony: A multielectrode study of cerebellar cortex activated by motor cortex. J. Neurophysiol. 86: 2489-2504.

Siebner, H.R. and Rothwell, J. 2003. Transcranial magnetic stimulation: New insight into representational cortical plasticity. Exp. Brain Res. 148: $1-16$.

Thach, W.T. 1998. What is the role of the cerebellum in motor learning and cognition? Trends Cogn. Sci. 2: 331-337.

Thompson, R.F. 2005. In search of memory traces. Annu. Rev. Psychol. 56: $1-23$.

Thompson, R.F., Bao, S., Chen, L., Cipriano, B.D., Grethe, J.S., Kim, J.J., Thompson, J.K., Tracy, J.A., Weninger, M.S., and Krupa, D.J. 1997. Associative learning. Int. Rev. Neurobiol. 41: 151-189.

van Alphen, A.M. and De Zeeuw, C.I. 2002. Cerebellar LTD facilitates but is not essential for long-term adaptation of the vestibulo-ocular reflex. Eur. J. Neurosci. 16: 486-490.

Van Der Meulen, J.P. and Gilman, S. 1965. Recovery of muscle spindle activity in cats after cerebellar ablation. J. Neurophysiol. 28: 943-957.

Voogd, J. 1995. Cerebellum. In: The rat nervous system (ed. G. Paxinos), pp. 309-350. Academic Press, San Diego.

Voogd, J. and Glickstein, M. 1998. The anatomy of the cerebellum. Trends Neurosci. 21: 370-375.

Wang, Y., Diao, R., Schalk, G., Wolpaw, J.R., and Chen, X.Y. 2003. Effects of H-reflex down-conditioning on GABAergic terminals on rat soleus motoneurons. Abstract Viewer/Itinerary Planner, Program No. 497.8. Society for Neuroscience, Washington, DC.

Wang, Y., Pillai, S.D., Wolpaw, J.R., and Chen, X.Y. 2004. Effects of H-reflex up-conditioning on GABAergic terminals on rat soleus motoneurons. Abstract Viewer/Itinerary Planner, Program No. 417.7. Society for Neuroscience, Washington, DC.

Wang, Y., Pillai, S.D., Wolpaw, J.R., and Chen, X.Y. 2006. Motor learning changes GABAergic terminals on spinal motoneurons in normal rats. Eur. J. Neurosci. 23: 141-150.

Whalen, P. and Pearson, K.G. 1997. Plasticity in reflex pathways controlling stepping in the cat. J. Neurophysiol. 78: 1643-1650.

Wolf, S.L., Segal, R.L., Heter, N.D., and Catlin, P.A. 1995. Contralateral and long latency effects of human biceps brachii stretch reflex conditioning. Exp. Brain Res. 107: 96-102.

Wolpaw, J.R. 1987. Operant conditioning of primate spinal reflexes: The H-reflex. J. Neurophysiol. 57: 443-459.

. 1997. The complex structure of a simple memory. Trends Neurosci. 20: 588-594.

. 2001. Spinal cord plasticity in the acquisition of a simple motor skill. In Spinal cord plasticity: Alterations in reflex function (eds. M.M. Patterson and J.W. Grau), pp. 101-125. Kluwer Academic, Boston. . 2002. Memory in neuroscience: Rhetoric versus reality. Behav. Cogn. Neurosci. Rev. 1: 130-163.

Wolpaw, J.R. and Carp, J.S. 1990. Memory trace in spinal cord. Trends Neurosci. 13: 137-142.

Wolpaw, J.R. and Herchenroder, P.A. 1990. Operant conditioning of H-reflex in freely moving monkeys. J. Neurosci. Methods 31: 145-152.

\section{Learning \& Memory}


Wolpaw, J.R. and Lee, C.L. 1989. Memory traces in primate spinal cord produced by operant conditioning of H-reflex. J. Neurophysiol.

61: $563-572$.

Wolpaw, J.R. and Tennissen, A.M. 2001. Activity-dependent spinal cord plasticity in health and disease. Annu. Rev. Neurosci. 24: 807-843.

Wolpaw, J.R., Braitman, D.J., and Seegal, R.F. 1983. Adaptive plasticity in the primate spinal stretch reflex: Initial development. $J$. Neurophysiol. 50: 1296-1311.
Wolpaw, J.R., Herchenroder, P.A., and Carp, J.S. 1993. Operant conditioning of the primate H-reflex: Factors affecting the magnitude of change. Exp. Brain Res. 97: 31-39.

Yeo, C.H. and Hesslow, G. 1998. Cerebellum and conditioned reflexes. Trends Cogn. Sci. 2: 322-330.

Received September 20, 2005; accepted in revised form January 13, 2006. 


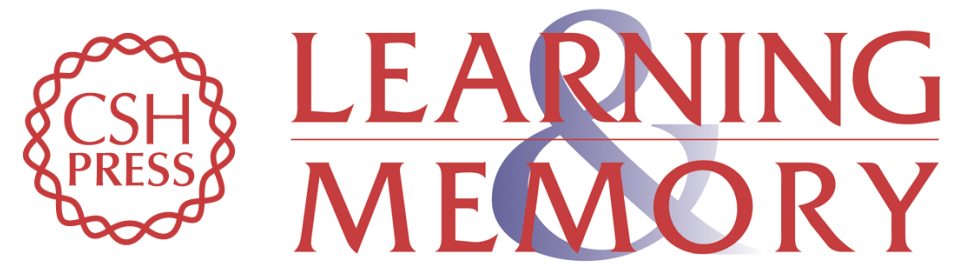

\section{The cerebellum in maintenance of a motor skill: A hierarchy of brain and spinal cord plasticity underlies $\mathrm{H}$-reflex conditioning}

Jonathan R. Wolpaw and Xiang Yang Chen

Learn. Mem. 2006, 13:

Access the most recent version at doi:10.1101/lm.92706

References This article cites 69 articles, 7 of which can be accessed free at:

http://learnmem.cshlp.org/content/13/2/208.full.html\#ref-list-1

License

Email Alerting Receive free email alerts when new articles cite this article - sign up in the box at the Service top right corner of the article or click here. 